\title{
Identifying Neurobiological Phenotypes Associated with Alcohol Use Disorder Severity
}

\author{
Eric D Claus*,', Sarah W Feldstein Ewing', Francesca M Filbey' ${ }^{2}$ Amithrupa Sabbineni' and \\ Kent E Hutchison ${ }^{1,3}$ \\ 'The Mind Research Network, Albuquerque, NM, USA; ${ }^{2}$ Center for BrainHealth, School of Behavioral and Brain Sciences, University of Texas at \\ Dallas, Dallas, TX, USA; ${ }^{3}$ Department of Psychology and Neuroscience, University of Colorado at Boulder, Boulder, CO, USA
}

\begin{abstract}
Although numerous studies provide general support for the importance of genetic factors in the risk for alcohol use disorders (AUDs), candidate gene and genome-wide studies have yet to identify a set of genetic variations that explain a significant portion of the variance in AUDs. One reason is that alcohol-related phenotypes used in genetic studies are typically based on highly heterogeneous diagnostic categories. Therefore, identifying neurobiological phenotypes related to neuroadaptations that drive the development of AUDs is critical for the future success of genetic and epigenetic studies. One such neurobiological phenotype is the degree to which exposure to alcohol taste cues recruits the basal ganglia, prefrontal cortex, and motor areas, all of which have been shown to have a critical role in addictive behaviors in animal studies. To that end, this study was designed to examine whether cue-elicited responses of these structures are associated with AUD severity in a large sample $(n=326)$ using voxelwise and functional connectivity measures. Results suggested that alcohol cues significantly activated dorsal striatum, insula/orbitofrontal cortex, anterior cingulate cortex, and ventral tegmental area. AUD severity was moderately correlated with regions involved in incentive salience such as the nucleus accumbens and amygdala, and stronger relationships with precuneus, insula, and dorsal striatum. The findings indicate that AUDs are related to neuroadaptations in these regions and that these measures may represent important neurobiological phenotypes for subsequent genetic studies.

Neuropsychopharmacology (201 I) 36, 2086-2096; doi:10.1038/npp.201 I.99; published online I5 June 20II
\end{abstract}

Keywords: craving; alcohol use disorder; phenotype; striatum; anterior cingulate cortex

\section{INTRODUCTION}

Although behavioral genetic research clearly supports the importance of genetic factors in the risk for alcohol use disorders (AUDs; Agrawal and Lynskey, 2008; Heath et al, 1999; Knopik et al, 2004), identifying loci that contribute to the heritability of alcohol dependence has proven to be difficult (Dick and Foroud, 2003; Bierut et al, 2010; Treutlein et al, 2009). While difficulty in characterizing the genetic basis of AUDs can be attributed to multiple factors, one important consideration is that alcohol-related phenotypes used in genetic studies often reflect broadly defined diagnostic categories that yield highly heterogeneous subgroups (Hutchison, 2010). To address this issue, behavioral scientists have advocated an alternative approach that emphasizes the characterization of specific, narrowly defined intermediate phenotypes, or endophenotypes (Cannon and Keller, 2006; Gottesman and Gould, 2003). Large-scale studies suggest that use of intermediate

*Correspondence: Dr ED Claus, The Mind Research Network, II01 Yale Boulevard NE, Albuquerque, NM 87I06, USA. Tel: + I 505925 4742; Fax: + I 505272 8002, E-mail: eclaus@mrn.org

Received 19 October 20 I0; revised 4 May 201 I; accepted 5 May 201 I phenotypes improve the ability to detect genetic factors that are associated with substance use disorders, including AUDs (Dick et al, 2006). Notably, recent reviews of this literature have called for efforts to develop intermediate neurobiological phenotypes, which could promote greater correspondence between human and animal models (Crabbe et al, 2010; Hutchison, 2010; Meyer-Lindenberg and Weinberger, 2006).

One potentially useful intermediate phenotype for AUDs is the degree to which exposure to alcohol cues recruits critical brain structures. This phenotype has been studied using animal models (eg, Rodd et al, 2004) as well as human neuroimaging studies (Filbey et al, 2008; George et al 2001; Hommer 1999; Wrase et al, 2007). One advantage of using cue-elicited changes in brain activation is the extensive animal literature, which provides a strong foundation for understanding the neurobiological mechanisms underlying the motivation to consume alcohol. The primary neural structures that have been identified as being critical for development of drug-seeking behavior in animal studies include the basolateral and central nucleus of the amygdala (Janak and Chaudhri, 2010; McBride, 2002), the medial prefrontal cortex (Carlson and Stevens, 2006), ventral tegmental area (VTA) (Stuber et al, 2008) and nucleus 
accumbens (NAc; Chaudhri et al, 2009; Knapp et al, 2009; Kelley, 2004), ventral pallidum (Harvey et al, 2002) and dorsal striatum (Wang et al, 2007), and the orbitofrontal cortex (OFC; Hansson et al, 2008). Alcohol and other drugrelated cues acquire motivational significance over repeated learning trials through the strengthening of connections among these regions (for reviews see, Koob and Volkow, 2010; Koob, 2008; Schultz, 2007). As a result, approach behaviors become more likely after presentation of alcohol and other drug-related cues.

The search for similar neural mechanisms in human beings was difficult until the advent of functional neuroimaging. Neuroimaging studies typically use tasks in which neural response to a drug cue is compared with response during a neutral, non-drug-related cue. For example, visual alcohol-related cues (eg, pictures of beer or wine) elicit increased responses in $\mathrm{NAc}$, anterior cingulate cortex (ACC), dorsolateral prefrontal cortex (DLPFC), OFC, hippocampus, and insula compared with neutral non-alcoholrelated cues (Lingford-Hughes et al, 2006; Myrick et al, 2004; Grusser et al, 2004; Ihssen et al, 2011). Presentation of the smell of one's favorite alcoholic beverage elicits enhanced response in NAc, dorsal striatum, occipital and parietal cortex, insula, and DLPFC (Kareken et al, 2004; Schneider et al, 2001). Finally, tastes of small amounts of alcoholic beverages have been found to elicit responses in ACC, DLPFC, OFC, insula, striatum, and thalamus (Filbey et al, 2008). Thus, in large part, human neuroimaging studies have replicated animal studies.

Although the above studies have provided information regarding the neural correlates of responses to alcohol cues, few of these studies (eg, Filbey et al, 2008) have reported associations of cue-elicited responses with severity of AUDs, and those that did often were limited to small sample sizes and a restricted range of alcohol use severity (eg, Filbey et al, 2008; George et al, 2001). This is an important question, as it is critical to identify neurobiological phenotypes (eg, BOLD response to alcohol taste cues) that are associated with clinical phenotypes (eg, loss of control over drinking) to better understand the development of AUDs. To address these issues, this study employs a large, diverse sample of heavy drinking individuals to identify the neurobiological phenotypes that are most strongly related to AUDs. In addition, this study examined demographic variables that are likely to influence these phenotypes, such as gender, treatment-seeking status, and smoking status. It was hypothesized that increased activation of ventral striatum, VTA, amygdala, OFC, DLPFC, and ACC would be associated with a greater history of alcohol exposure (ie, years of regular drinking) and greater AUD severity. We also hypothesized that BOLD response to alcohol cues would be greater in men, smokers, and treatment seekers.

\section{MATERIALS AND METHODS}

\section{Participants}

In all, 326 heavy drinking individuals (98 women; $30 \%$ ) were included in this study. With institutional IRB approval, participants were recruited from the greater Albuquerque metropolitan region through advertisements placed in local print, online media, and radio advertisements to participate in two different translational studies evaluating alcohol use across heavy drinking treatment-seeking and non-treatment-seeking samples. This study focuses on the baseline relationship between alcohol use symptoms and brain response, and is therefore limited to the baseline behavioral and neuroimaging assessments from both studies. Treatment-seeking participants had not initiated psychosocial or pharmacological treatment at the time of the scanning session. During the baseline assessments, participants completed questionnaire data and a neuroimaging session that lasted approximately $2 \mathrm{~h}$. Participants were compensated US $\$ 120$ for their efforts. To participate in these studies, participants had to drink at least 5 or more drinks per drinking occasion for men (4 or more for women) at least five times in the past month. Participants were excluded if they reported a history of severe alcohol withdrawal, previous injury to the brain, or loss of consciousness for more than $5 \mathrm{~min}$. In addition, all women were required to test negative on a pregnancy test administered before entering the MRI suite. All participants were required to have a breath alcohol concentration of 0.00 as measured by a breathalyzer before their participation in the scanning and assessment session and could not be in need of medical detoxification, as assessed by a score greater than eight on the Clinical Institute Withdrawal Assessment of Alcohol Scale, Revised (CIWA-Ar; Sullivan et al, 1989).

\section{Materials}

To evaluate demographic variables, alcohol use and severity, and other substance use (including tobacco), the following questionnaires were administered: a demographics questionnaire, the Alcohol Use Disorder Identification Test (AUDIT) (Babor et al, 2001), the Alcohol Dependence Scale (ADS) (Skinner and Horn, 1984), the Time-Line Follow-Back (TLFB) for quantity and frequency of alcohol, cigarettes, and marijuana (Sobell and Sobell, 1992), the Failed Control subscale of Impaired Control Scale (ICS) (Heather et al, 1998; Heather et al, 1993), and a questionnaire to assess years of regular drinking.

\section{Alcohol Taste Cue Task}

To measure cue-elicited responses to alcohol, we used a task described previously in our lab (Filbey et al, 2008; Hutchison et al, 2008). Participants received small amounts of two different beverages, pseudorandomly presented through Teflon tubing to the participant while they were in the scanner by a computer-controlled gustometer. The two beverages included small amounts (eg, $1 \mathrm{ml}$ ) of their preferred alcoholic beverage (eg, wine, alcohol, mixed drinks) alternated with a control beverage (litchi juice) that was selected for its appetitive and novel qualities. Each trial within the task began with a 'Ready' prompt for $2 \mathrm{~s}$ that designated the start of the trial, followed by taste cue presentation for $24 \mathrm{~s}$, during which participants were instructed to Taste Alcohol (or Juice; seconds 1-10 and 12-22) and Swallow (seconds 10-12, 22-24). The taste cue presentation was followed by a washout period during which no taste stimuli were presented and participants viewed the word Rest for $16 \mathrm{~s}$. Participants completed six trials of each tastant in each of two 9 min runs. 


\section{Image Acquisition}

All MRI data was collected on a 3T Siemens Trio (Erlangen, Germany) whole body scanner. Participants were placed in the scanner and a piece of tape was placed across the forehead to serve as feedback for movement reduction.

Before the acquisition of anatomical scans, localizer scans were acquired. An echo-planar gradient-echo pulse sequence $\left(\mathrm{TR}=2000 \mathrm{~ms}, \mathrm{TE}=29\right.$, flip angle $\left.=75^{\circ}\right)$ was acquired with an 8-channel head coil, and images were acquired parallel to the ventral surface of a participant's OFC to reduce signal dropout and distortion in this region (Deichmann et al, 2003). Each volume acquired consisted of 33 axial slices $\left(64 \times 64\right.$ matrix, $3.75 \times 3.75 \mathrm{~mm}^{2}, 3.5 \mathrm{~mm}$ thickness, $1 \mathrm{~mm}$ gap). In addition, a high resolution T1-weighted MP-RAGE anatomical image was acquired $\left(\mathrm{TR}=2530 \mathrm{~ms}, \mathrm{TE}=1.64 \mathrm{~ms}\right.$, flip angle $=7^{\circ}, 192$ sagittal slices, $256 \times 256$ matrix, slice thickness $=1 \mathrm{~mm}$, no gap) for each participant.

\section{Image Analysis}

All analyses were completed using tools from the FMRIB Software Library (FSL). The first seven volumes of each functional run were discarded to allow the magnet to reach steady state. Motion Correction using FMRIB's Linear Image Registration Tool (Jenkinson et al, 2002) was used to realign images to the first volume within a run. Images were deskulled using BET, spatially smoothed with an $8 \mathrm{~mm}$ full-width half-max Gaussian kernel, temporally filtered using a high-pass filter of $100 \mathrm{~s}$, and grand mean intensity normalized; all of these steps were performed using FMRIB Expert Analysis Tool (FEAT; Smith et al, 2004). Regressors of interest were created for the following conditions: alcohol cue, alcohol baseline, alcohol urge, juice cue, juice baseline, and juice urge, according to the timing scheme used in previous studies (Filbey et al, 2008; Hutchison et al, 2008). The primary contrast of interest reported in this study compared the alcohol cue $v s$ juice cue. Statistical analyses were performed using the general linear model as implemented in FEAT. Customized square waveforms representing the condition of interest and the duration of stimulus presentation were convolved with a double gamma hemodynamic response function. Time-series analyses were conducted using FMRIB Improved Linear Model (Woolrich et al, 2004) with local autocorrelation estimation. This firstlevel analysis generated parameter estimates for each condition of interest, and contrast maps were computed for each participant. Contrast maps were registered to the Montreal Neurological Institute (MNI) 152-brain template using a two-step registration process with FMRIB Linear Image Registration Tool (FLIRT; Jenkinson et al, 2002). First, an average EPI image was registered to the participant's high-resolution anatomical image. Each participant's high-resolution T1-weighted image was then registered to the MNI 152-brain template. Finally, contrast maps were registered to the MNI 152-brain template using parameters from the previous registration steps.

Individual runs were combined within subjects using a fixed-effects model. The results from the second-level analyses were then used in a third-level group analysis using FMRIB Local Analysis of Mixed Effects (Woolrich et al,
2004) stage 1 only. Before computing group level statistics, all second-level contrast images were registered to the MNI template using parameters from the two-step registration process described above. For examinations of continuous variables (ie, AUDIT, ADS, ICSFC, years of regular alcohol use), we used linear regression to determine the correlation between the alcohol scale of interest and the contrast map of alcohol $v s$ juice. To examine group differences as a function of gender, smoking status, and treatment-seeking status, we performed independent group $t$-tests on the contrast maps. Participants were considered smokers if they reported any cigarette smoking in the past 60 days. All group maps were masked to only examine gray matter. To protect against multiple comparison problems, group level statistical maps were thresholded using Gaussian random field theory (Worsley et al, 1992, 1996) as implemented in cluster-based thresholding in FSL. For each analysis, we used a voxelwise threshold of $z=3.09$ and cluster threshold of $p<0.05$ (In addition, all analyses were repeated using a voxelwise threshold of $z=2.3$ and cluster level significance level of $p<0.05$, to show the extent of the activation at standard statistical thresholds. Images for each of these analyses can be found in Supplementary Figures S1-S6.) In addition, the main effect analysis comparing alcohol to litchi was thresholded using a voxelwise threshold of $z=8, p<7 \times 10^{-16}$.

\section{Regions of Interest Analysis}

In addition to our whole brain analysis, we also examined bilateral regions of interest (ROI) previously shown to be associated with enhanced response to alcohol cues to determine if these regions were associated with AUD severity including amygdala, NAc, VTA, DLPFC, OFC, and ACC. For the OFC, amygdala, and NAc ROIs, we used the Harvard Oxford probabilistic atlas in FSL to identify voxels within each ROI. The DLPFC ROI was a sphere with a radius of $5 \mathrm{~mm}$ around the DLPFC peak reported by Tapert et al. (2004). VTA was defined using a sphere with a radius of $2.5 \mathrm{~mm}$ around the coordinate $x=0, y=-16$, and $z=-8$ (D'Ardenne et al, 2008). ACC was defined using a sphere with a $5 \mathrm{~mm}$ radius around the maximum response in ACC $(x=-6, y=30, z=14)$ reported by Filbey et al. (2008). ROIs were then used as masks in the group level and covariate analyses; in each case, statistical maps were thresholded using Gaussian random field theory (Worsley et al, 1992, 1996) as implemented in cluster-based thresholding in FSL (voxel threshold $z=2.3 / p=0.01$; cluster threshold $p<0.05)$.

\section{RESULTS}

As shown by responses on the SCID and TLFB (see Table 1), this sample provided a wide range of AUD severity. As expected, treatment seekers showed higher scores on measures related to AUD severity such as the AUDIT ( $t(322)=$ $12.22, p<0.001)$, the ADS $(t(323)=9.74, p<0.001)$, the ICSFC scale $(t(324)=13.78, p<0.001)$, and years of regular drinking $(t(324)=14.44, p<0.001)$. A greater proportion of non-treatment seekers smoked marijuana $\left(\chi^{2}=12.96\right.$, $p<0.001)$ than treatment seekers, but the two groups did not differ on cigarette smoking $\left(\chi^{2}=2.74, p=0.09\right)$. As 
Table I

Sample Characteristics

\begin{tabular}{|c|c|c|c|c|}
\hline \multirow[t]{3}{*}{ Gender } & \multicolumn{4}{|c|}{ Treatment seeking } \\
\hline & \multicolumn{2}{|c|}{ Yes } & \multicolumn{2}{|c|}{ No } \\
\hline & Male & Female & Male & Female \\
\hline$n$ & 96 & 51 & 130 & 49 \\
\hline Age & $39.0(9.1)$ & $40.6(8.8)$ & $26.1(4.6)$ & $24.3(2.5)$ \\
\hline ADS total & I7.| (8.4) & $19.3(9.0)$ & $10.2(5.8)$ & $9.4(5.7)$ \\
\hline AUDIT & $23.3(6.4)$ & $24.6(7.7)$ & $15.6(6.0)$ & | $3.6(5.4)$ \\
\hline ICS-FC & I5.| (6.7) & I3.9 (7.6) & $26.1(8.1)$ & $26.6(7.8)$ \\
\hline Yrs drinking & $20.2(9.8)$ & $19.4(9.6)$ & $8.4(5.5)$ & $6.6(3.5)$ \\
\hline Avg. drinks & $9.6(5.5)$ & $8.0(4.3)$ & $6.3(2.4)$ & $4.9(2.1)$ \\
\hline Drinking days (30) & $20.0(8.3)$ & $19.9(7.8)$ & $16.0(6.5)$ & $\mid 4.1$ (6.1) \\
\hline \% Cig smokers & 39.6 & 51.0 & 35.4 & 34.7 \\
\hline Avg. cigs & I I.I (7.3) & |2.| (8.2) & I $1.7(7.5)$ & $7.6(5.9)$ \\
\hline \% MJ smokers & 29.5 & 20.0 & 46.5 & 45.8 \\
\hline \% Days smoked MJ & $24.1(25.7)$ & $26.5(38.7)$ & $30.0(35.6)$ & $38.0(38.8)$ \\
\hline CIWA range (mean) & $0-7(1.56)$ & $0-5(1.68)$ & - & - \\
\hline
\end{tabular}

ADS_Alcohol Dependence Scale; AUDIT_Alcohol Use Disorder Identification Test; ICS-FC — Impaired Control Scale, Failed Control subscale; Yrs drinking — number of years of regular drinking; Avg. drinks - average number of standard drinks consumed per drinking day; drinking days (30) — number of drinking days in the past 30 days; \% Cig smokers — percentage of participants who reported smoking in the past 60 days; Avg. cigs - number of cigarettes smoked per smoking day; \% MJ smokers - percentage of participants who reported smoking marijuana at least one time per month; \% days smoked MJ-percentage of days that marijuana was smoked.

Means (SD) for measures collected during the baseline questionnaire session.

Table 2 Correlations between Alcohol Severity and Exposure Variables

\begin{tabular}{lcccc}
\hline & AUDIT & ADS & ICS-FC & Yrs drinking \\
\hline AUDIT & - & 0.82 & 0.78 & 0.44 \\
ADS & - & - & 0.70 & 0.34 \\
ICS-FC & - & - & - & 0.39 \\
\hline
\end{tabular}

ADS —Alcohol Dependence Scale; AUDIT—Alcohol Use Disorder Identification Test; ICS-FC - Impaired Control Scale, Failed Control subscale; Yrs drinking - number of years of regular drinking. All p's $<0.0000$ l.

expected, our covariates of interest were highly intercorrelated (see Table 2). Across all participants, 51\% received beer, $40 \%$ received spirits, and $9 \%$ received wine as the alcohol-containing beverage during the taste task.

\section{Main Effects of Alcohol Cues vs Control Cues}

As seen in Figure 1, across all participants, the contrast of alcohol $v s$ juice shows a difference that encompasses one large cluster that includes the entire striatum, thalamus, medial frontal cortex (ACC, dorsomedial PFC, supplementary motor area), brainstem, bilateral OFC, bilateral insula, amygdala, and cerebellum. Peak detection algorithms showed that peaks of activation differences appear in ACC (BA 24), right lateral OFC, bilateral anterior insula, bilateral amygdala, bilateral caudate head, thalamus, putamen, VTA, posterior cingulate cortex, and cerebellum (see Figure 1 and Table 3). In addition, in the ROI analysis, we found significant effects in all ROIs, except right DLPFC (see Table 4).

\section{Covariate Analyses}

Audit. In the whole brain analysis, positive correlations between the AUDIT and the contrast of alcohol $v s$ juice emerged in precuneus, posterior insula, posterior cingulate cortex, globus pallidus, and putamen (see Figure $2 \mathrm{a}$ and Table 3). ROI analyses showed positive significant relationships with AUDIT scores in left NAc, left OFC, left DLPFC, and right amygdala (see Table 4).

Alcohol dependence scale. In the whole brain analysis, positive correlations between the ADS and the contrast of alcohol $v s$ juice emerged in cuneus, precentral gyrus, and fusiform gyrus $(z>3.09, p<0.05)$ (see Figure $2 \mathrm{~b}$ and Table 3 ). ROI analyses showed significant positive correlations with ADS in ACC, left DLPFC, left OFC, bilateral NAc, and right amygdala (see Table 4 ).

ICS - failed control. Our whole brain analysis showed that failures in control over drinking were positively associated with the alcohol-juice contrast in the caudal portion of the ACC extending into the supplementary motor area, bilateral pre/postcentralgyrus, bilateral insula, bilateral putamen, thalamus, and globus pallidus, parahippocampal gyrus, precuneus, and brainstem (see Figure 2c and Table 3; $z>3.48, p<0.05)$. ICSFC showed significant positive relationships with several ROIs including bilateral amygdala, left DLPFC, and left NAc (see Table 4).

Years of regular drinking. Whole brain analyses of the correlation between self-reported years of regular drinking and BOLD responses in the alcohol-juice contrast showed significant relationships in precuneus/lateral occipital cortex, and cuneus (see Figure $2 \mathrm{~d}$ and Table 3). ROI analyses with years of regular drinking showed significant effects bilateral DLPFC and left NAc (see Table 4).

Multiple regression analyses. Because all our independent variables measuring severity of AUDs were highly correlated, we examined the independent influence of each variable to the overall alcohol $v s$ juice contrast by entering each variable in a multiple regression analysis in which each independent variable was orthogonalized with respect to each other intravenously. In the multiple regression analysis, AUDIT showed no significant effects that met cluster level thresholding. In contrast, ICSFC showed a positive relationship with the alcohol-litchi contrast in a cluster that included right lateralized central operculum/ insula, precentral gyrus, and putamen. ADS showed a positive relationship in inferior precuneus/cuneus. Finally, years of regular drinking showed a positive relationship with inferior and superior precuneus and lateral occipital cortex. 


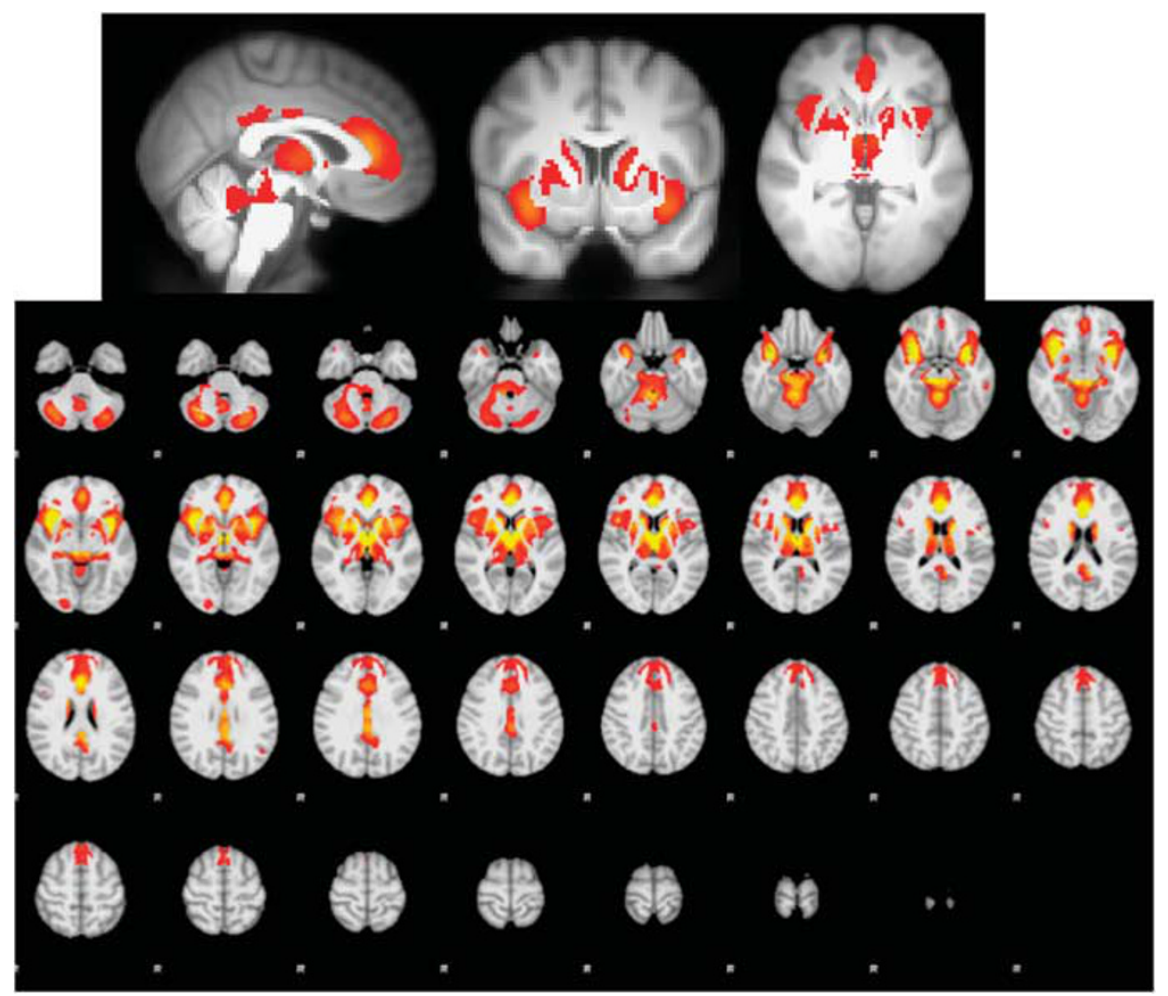

Figure I Main effect of blood oxygen level-dependent (BOLD) response to alcohol cues-litchi cues, cluster corrected at $z>8, p<0.05$, for visualization purposes. The comparison of alcohol cues to litchi cues showed significant differences in anterior and posterior cingulate cortex, dorsal striatum, insula, thalamus, and brainstem.

\section{Group Level Comparisons}

Gender. No significant differences were found when comparing responses to alcohol vs litchi across men and women in either whole brain analysis. However, in the ROI analysis, men showed enhanced response in the left amygdala compared with women in the alcohol-litchi contrast. This relationship remained even after controlling for ADS scores.

Smoking. No significant differences emerged between cigarette smokers and non-smokers in the alcohol-juice contrast in the whole brain analysis. However, the ROI analysis showed differences in bilateral NAc, such that nonsmokers had a greater difference between alcohol and litchi. However, given that non-smokers scored higher on AUD severity than smokers, we covaried ADS scores and found that the NAc findings were no longer significant, suggesting that AUD severity was driving the effect.

Treatment-seeking status. Compared with non-treatment seekers, treatment-seeking subjects showed significantly greater response in precuneus, middle temporal gyrus, inferior temporal gyrus, and SMA. In contrast, nontreatment seekers did not show any significantly greater responses in the contrast of alcohol-litchi compared with treatment seekers (see Figure 2e and Table 3). In the ROI analysis, treatment seekers showed greater response than non-treatment seekers in left DLPFC, left NAc, and left amygdala (see Table 4).

\section{DISCUSSION}

Exposure to the taste of alcohol as compared with an appetitive control taste cue resulted in widespread response throughout regions important for incentive motivation (Arana et al, 2003; McClure et al, 2003; Robinson and Berridge, 2003) and planned motor behavior (Rushworth et al, 2004). Specifically, we found maximal differences in ACC, bilateral OFC/insula, dorsal striatum, amygdala, thalamus, VTA, and cerebellum when comparing neural responses to the delivery of alcohol and litchi juice. Additional differences appeared in bilateral ventral striatum and left DLPFC in the ROI analyses. These findings were not surprising, given the myriad studies investigating cueelicited responses to alcohol that have shown similar effects (Filbey et al, 2008; George et al 2001; Kareken et al 2004; Wrase et al, 2007). Although previous studies have primarily focused on ventral striatal responses to alcohol cues, this study suggests that dorsal striatum has an integral role in addictive behavior Vollstadt-Klein et al, 2010). The dorsal striatum contributes significantly to habit learning (Belin et al, 2009; Costa, 2007), an expected consequence of repeated ingestion of rewarding substances such as alcohol (Wise, 2009; Koob and Volkow, 2010). Dorsal striatal circuits project and interact with supplementary motor areas involved in motor responses and planning. Specifically, it is believed that GABAergic cell bodies in the dorsal striatum project to the globus pallidus, and activation of these cells results in a disinhibition of the thalamus, allowing sensory input to flow to motor control regions of 
Table 3 Cluster Sizes and Locations of Significant Clusters in all Neuroimaging Analyses

\begin{tabular}{|c|c|c|c|c|c|c|c|c|}
\hline Contrast & Brain region & BA & $\operatorname{Max} z$ & Mean $r(\max r)$ & Voxels & $x$ & $y$ & $\mathbf{z}$ \\
\hline \multirow[t]{7}{*}{ Main effect } & Inferior frontal gyrus (L) & 13 & 11.7 & - & 3160 & -34 & 10 & -14 \\
\hline & Extra-nuclear (R) & 13 & 12 & - & 1224 & 36 & 12 & -12 \\
\hline & Anterior cingulate $(L)$ & 24 & 12.6 & - & 861 & 0 & 30 & 10 \\
\hline & Cingulate gyrus (L) & 23 & 9.27 & - & 273 & 0 & -16 & 28 \\
\hline & Thalamus $(L)$ & & 8.62 & - & 36 & -20 & -20 & 10 \\
\hline & Cerebellum (L) & & 8.08 & - & 2 & -26 & -70 & -34 \\
\hline & Caudate (R) & & 8.03 & - & I & 18 & -8 & 22 \\
\hline \multirow[t]{3}{*}{ AUDIT } & Precuneus (R) & 31 & 4.46 & $0.20(0.24)$ & 1151 & 16 & -58 & 22 \\
\hline & Insula (R) & 13 & 4.52 & $0.20(0.24)$ & 878 & 48 & -34 & 24 \\
\hline & Paracentral lobule (R) & 5 & 4.34 & $0.19(0.23)$ & 781 & 8 & -36 & 46 \\
\hline \multirow[t]{4}{*}{ ADS } & Cuneus (L) & 7 & 4.73 & $0.19(0.24)$ & 2104 & -6 & -72 & 32 \\
\hline & Precentral gyrus (L) & 6 & 4.8 & $0.19(0.24)$ & 1679 & -62 & 4 & 6 \\
\hline & Fusiform gyrus (L) & 19 & 4.78 & $0.19(0.25)$ & 948 & -22 & -66 & -10 \\
\hline & Cerebellum (L) & & 3.89 & $0.18(0.21)$ & 397 & -22 & -56 & -38 \\
\hline \multirow[t]{6}{*}{ ICSFC $^{a}$} & Insula (R) & 13 & 4.91 & $0.21(0.25)$ & 2362 & 48 & -36 & 22 \\
\hline & Inferior parietal lobule $(L)$ & 40 & 5.17 & $0.21(0.25)$ & 2255 & -48 & -38 & 28 \\
\hline & Precuneus (L) & 7 & 5.14 & $0.21(0.26)$ & 1494 & -12 & -52 & 48 \\
\hline & Brainstem $(L)$ & & 4.77 & $0.21(0.24)$ & |34| & -10 & -24 & -30 \\
\hline & Parahippocampal gyrus (R) & 35 & 5.23 & $0.21(0.25)$ & 987 & 20 & -20 & -12 \\
\hline & Medial frontal gyrus $(\mathrm{L})$ & 6 & 4.76 & $0.21(0.25)$ & 736 & -8 & -2 & 54 \\
\hline \multirow{2}{*}{ Tx>non-Tx } & Inferior temporal gyrus (L) & 20 & 4.26 & - & 854 & -44 & -14 & -28 \\
\hline & Medial frontal gyrus $(\mathrm{L})$ & 6 & 4.05 & - & 343 & -4 & -8 & 54 \\
\hline
\end{tabular}

AUDIT_Alcohol Use Disorder Identification Test; ADS_Alcohol Dependence Scale; ICS-FC_-Impaired Control Scale, Failed Control subscale;

Yrs drinking — number of years of regular drinking; Tx — treatment seeking; non-Tx — on-treatment seeking.

amages thresholded at $z>3.48, p<0.05$ cluster corrected. Mean (max) $r$-mean (max) correlation coefficient within the significant cluster.

frontal cortex (Alexander and Crutcher, 1990; Alexander et al, 1990; Wickens, 1997). Given that we found both dorsal striatum and presupplementary motor area in our main contrast, this may suggest that functional connectivity between these regions is enhanced as a result of habit learning. Future studies are needed to investigate the functionally connected networks that contribute to craving responses.

In addition to the main contrast of alcohol and litchi, we were also interested in how these differences correlated with measures of AUD severity. Commonalities among the correlations between clinical measures and brain responses included significant relationships with signal change in left NAc, left DLPFC, and amygdala in the ROI analyses. Similar correlation patterns with severity measures also occurred in the precuneus and globus pallidus, insula, parahippocampal gyrus, and ACC/SMA in the whole brain analyses. The amygdala and NAc are two important structures for assigning value to emotional stimuli, and left DLPFC may be important for directing attention towards rewarding stimuli (Savine and Braver, 2010). The basolateral amygdala and NAc, along with frontal cortex, are part of a final common pathway for cue-elicited craving and relapse (Kalivas and Volkow, 2005; Koob and Volkow, 2010).

Although the amygdala/NAc results confirm previous models and findings, this study adds to the previous literature by suggesting a prominent role of precuneus and insula in cue reactivity in more severe AUDs. While previous studies have shown evidence of the role of precuneus in cue reactivity (Tapert et al, 2004; Myrick et al, 2008), structures within the mesolimbic dopamine system have received the most focus. However, the insula has received increasing 
Table 4 Relationships between ROls and Clinical Assessment Variables

\begin{tabular}{|c|c|c|c|c|c|c|c|}
\hline Measure & Brain region & $\operatorname{Max} z$ & Mean $r(\max r)$ & Voxels & $x$ & $y$ & $z$ \\
\hline \multirow[t]{7}{*}{ Main effect } & ACC & 12.3 & - & 81 & -2 & 28 & 12 \\
\hline & Left amygdala & 9.68 & - & 299 & -32 & 0 & -16 \\
\hline & Left DLPFC & 3.98 & - & 14 & -18 & 22 & 50 \\
\hline & Left OFC & 9.99 & - & 997 & -30 & 10 & -18 \\
\hline & Left NAc & 7.61 & - & 48 & -8 & 6 & -4 \\
\hline & Right NAc & 7.31 & - & 57 & 10 & 10 & -4 \\
\hline & VTA & 7.89 & - & 7 & 0 & -16 & -6 \\
\hline \multirow[t]{2}{*}{ AUDIT } & Left DLPFC & 3.22 & $0.15(0.178)$ & | | & -18 & 18 & 50 \\
\hline & Left OFC & 3.55 & $0.15(0.189)$ & 122 & -36 & 32 & 0 \\
\hline \multirow[t]{8}{*}{ ADS } & $\mathrm{ACC}$ & 2.43 & $0.13(0.135)$ & 2 & -8 & 26 & 12 \\
\hline & Left DLPFC & 3.11 & $0.15(0.172)$ & 19 & -18 & 18 & 52 \\
\hline & & 2.37 & $0.13(0.131)$ & 2 & -26 & 18 & 50 \\
\hline & Left OFC & 3.59 & $0.15(0.185)$ & 172 & -40 & 30 & -2 \\
\hline & Left NAc & 3.15 & $0.15(0.174)$ & 46 & -14 & 16 & -6 \\
\hline & Right amygdala & 3.15 & $0.14(0.174)$ & 32 & 24 & -10 & -10 \\
\hline & Right NAc & 2.34 & $0.13(0.13)$ & I & 8 & 18 & -2 \\
\hline & & 2.32 & $0.13(0.128)$ & I & 6 & 14 & -4 \\
\hline ICSFC & Left amygdala & 3.89 & $0.16(0.207)$ & 223 & -22 & -10 & -12 \\
\hline \multirow[t]{2}{*}{ Non-smoker > smoker } & Left NAc & 3.74 & - & 39 & -12 & 10 & -6 \\
\hline & Right NAc & 3.29 & - & 45 & 12 & 12 & -6 \\
\hline \multirow[t]{3}{*}{$T x>$ non- $T x$} & Left amygdala & 3.54 & - & 47 & -30 & -8 & -20 \\
\hline & Left DLPFC & 3.65 & - & 31 & -18 & 18 & 50 \\
\hline & Left NAc & 3.79 & - & 56 & -12 & 6 & -10 \\
\hline
\end{tabular}

AUDIT_Alcohol Use Disorder Identification Test; ADS_Alcohol Dependence Scale; ICS-FC_Impaired Control Scale, Failed Control subscale; Yrs drinking - number of years of regular drinking; Tx - treatment seeking; non-Tx - non-treatment seeking.

Images were thresholded at $z>2.3, p<0.05$ cluster corrected. Mean (max) $r$-mean (max) correlation coefficient within the significant cluster.

amounts of attention in the addiction literature since reports that damage to this region resulted in reduced levels of craving in smokers (Naqvi et al, 2007). The correlations of the insula with AUD severity measures suggests a potential increase in processing of interoceptive cues triggered by cue presentation (Bechara 2005; Critchley, 2005; Goldstein et al, 2009; Gray and Critchley, 2007; Lovero et al, 2009; Naqvi and Bechara, 2009; Paulus, 2007). The precuneus is functionally connected to the insula and also portions of the dorsal striatum (Marguiles et al, 2009), and these findings suggest that this functional circuit may be enhanced in individuals with more severe AUDs. Again, because we did not specifically investigate functional connectivity, this speculation must be tested in future studies. Regardless, given the role of the precuneus in maintaining attention and mental imagery (Cavanna and Trimble, 2006), the findings in this study suggest that this region may be important in automatic attentional biases towards alcohol-related cues (Sharma et al, 2001; Stetter et al, 1995; Stormark et al, 1997).

In addition to severity of use, we examined factors that have been previously shown to influence subjective craving 


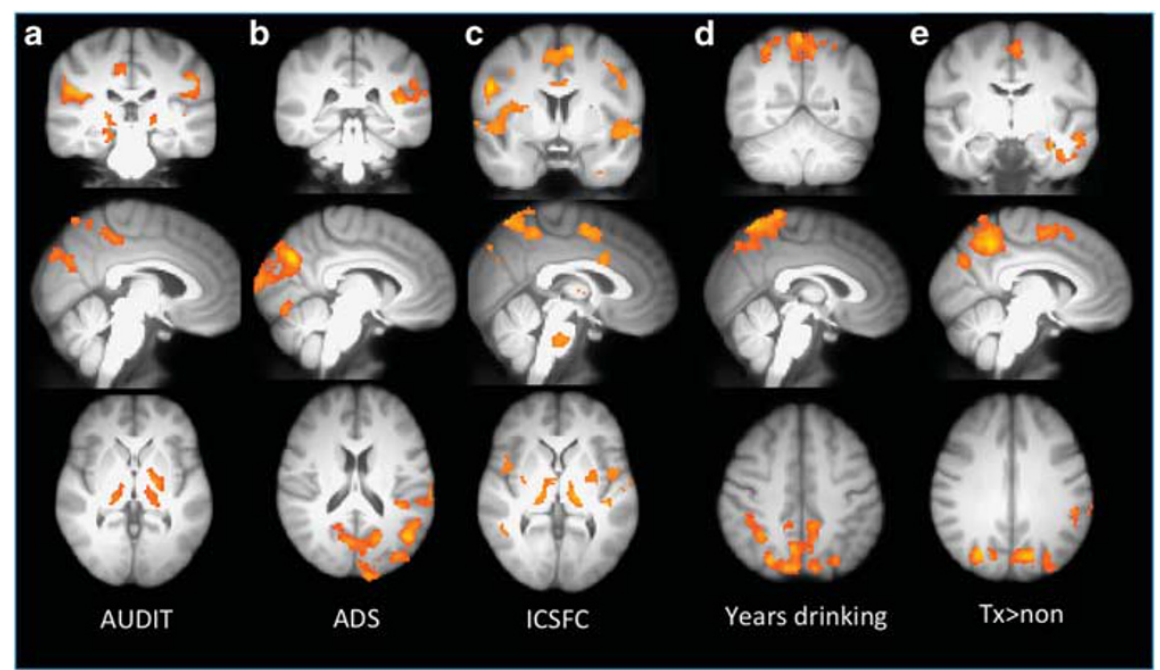

Figure 2 Correlation of blood oxygen level-dependent (BOLD) response with measures of alcohol abuse severity. Examination of the correlation between the alcohol-litchi contrast image and four measures of alcohol use severity/alcohol exposure suggests that craving responses for alcohol cues engage regions associated with habit learning and motor control in more experienced drinkers and those who experience more problems as a result of alcohol use (a-d). (e) Differences in the alcohol vs litchi contrast between treatment seekers and non-treatment seekers. Treatment seekers showed greater differences between the two conditions in the precuneus, SMA, compared with non-treatment seekers. All images are threshold at $z=3.09$, cluster corrected $p<0.05$, except where noted. (a) AUDIT_Alcohol Use Disorder Identification Test; (b) ADS—Alcohol Dependence Scale; (c) ICS-FC - Impaired Control Scale, Failed Control subscale; (d) years of regular drinking; and (e) treatment seeking vs non-treatment seeking.

or neural responses to cues. These included factors such as gender, smoking status, and treatment-seeking status. The comparison of men and women revealed significant differences in the left amygdala, such that men had greater responses than women. These findings are consistent with previous studies that suggest men have a stronger affective response to rewarding stimuli (Hamann, 2005), and suggest that men found the alcohol more rewarding than the women. Smoking status influenced responses in bilateral NAc, but this effect was likely due to the fact that nonsmokers had more severe AUDs, as measured by the AUDIT and ADS. When examining treatment seekers compared with non-treatment seekers, we found activation differences in regions that were largely consistent with those observed in the AUDIT and ICSFC analyses. In addition, treatmentseeking subjects showed greater response in ventral striatum in the ROI analysis. These findings are not surprising given that treatment seekers showed more severe dependence and more years of drinking compared with non-treatment seekers. Unexpectedly, treatment seekers actually showed greater activation of DLPFC than nontreatment seekers, a finding that is inconsistent with a previous review of cue-elicited craving that report DLPFC and OFC only in non-treatment seekers (Wilson et al, 2004). One potential difference between this study and those cited by Wilson et al (2004) is the number of subjects included in each analysis. Many previous studies of cue-elicited craving have used very small sample sizes, which may have limited the ability to detect smaller effects or resulted in spurious findings. However, this study includes a very large sample of treatment- and non-treatment-seeking subjects, and thus power and sample representativeness are not likely issues. These results suggest that frontally mediated craving responses are enhanced in individuals seeking treatment for alcohol dependence, and it may be possible that alcohol and associated cues usurp frontal control systems in the later stages of alcohol dependence that are used to obtain or plan out future drug seeking behavior. Alternatively, these frontal regions may be preferentially recruited in treatment seekers because they are actively trying to control urges to drink, given that these individuals participated in a study advertising treatment for alcohol dependence. Future studies are needed to adjudicate between these alternative hypotheses.

\section{Limitations and Conclusions}

Although this study provides a sufficiently powered investigation into the neural correlates of cue-induced craving and the relationship with severity of alcohol problems, it is important to consider the findings in light of the following limitations. First, to participate, individuals were required to abstain from drinking. While none of our participants were in need of medical detoxification (as determined by the CIWA), it would be logical to assume that during the scan session, many may have experienced some level of withdrawal that may have contributed to the observed patterns of activation. As many studies with nicotine have shown, withdrawal can influence BOLD responses in ACC, striatum, posterior cingulate, and DLPFC (McBride et al, 2006; McClernon et al, 2008), suggesting that many of our correlations with severity may also be modulated by withdrawal state. Future studies will be needed that explicitly tease apart the effects of acute withdrawal and AUD severity on craving responses. Second, our treatment-seeking participants used less marijuana than our non-treatment participants, a potential confound when interpreting the results of this study. For example, recent cannabis use has been shown to decrease frontal responses under conditions of stress ( $\mathrm{Li}$ et al, 2005), and the differences in marijuana 
use between our treatment and non-treatment samples may have influenced frontal responses during the taste task.

In conclusion, this study has identified the core neural substrates that underlie responses to alcohol cues and identified specific regions that are related to the progression of alcohol abuse severity. Across all participants, alcohol cues, compared with a juice cue, significantly activated regions important for motivated behavior such as lateral OFC, anterior cingulate, ventral striatum, caudate, and putamen. In addition, AUD severity and years of regular drinking were correlated with regions involved in sensorimotor processing such as the precuneus and supplementary motor area. Overall, these results suggest that as individuals progress along a continuum of AUD severity, regions involved in motor behavior in addition to affective processing, become more potently engaged in response to alcohol-related cues. Greater recruitment of motor circuits may correspond with an increasing lack of control over alcohol use. The findings suggest that measures of functional changes in these regions may represent an important phenotype for genome-wide studies that are designed to identify genetic variation that underlies the progression of neuroadaptations in these regions. Finally, it is important to note that these phenotypes may also represent important targets for the development of new treatment approaches. For example, medications that disrupt the connection between the striatum and motor areas may be particularly useful for relapse prevention. Ultimately, these neurobiological phenotypes may eventually be useful for identifying genetic variations or brain measures that predict response to treatment (see Hutchison, 2010).

\section{ACKNOWLEDGEMENTS}

This research was supported by grants from the National Institute on Alcoholism and Alcohol Abuse (AA012238 and AA014886) to $\mathrm{KH}$.

\section{DISCLOSURE}

The authors declare that, except for income received from their primary employer, no financial support has been received from any individual or corporate entity over the past 3 years for research or professional service and there are no financial holdings that could be perceived as constituting a potential conflict of interest.

\section{REFERENCES}

Agrawal A, Lynskey MT (2008). Are there genetic influences on addiction: evidence from family, adoption and twin studies. Addiction 103: 1069-1081.

Alexander GE, Crutcher MD (1990). Functional architecture of basal ganglia circuits: neural substrates of parallel processing. Trends Neurosci 13: 266-271.

Alexander GE, Crutcher MD, DeLong MR (1990). Basal gangliathalamocortical circuits: parallel substrates for motor, oculomotor, 'prefrontal' and 'limbic' functions. Prog Brain Res 85: 119-146.

Arana FS, Parkinson JA, Hinton E, Holland AJ, Owen AM, Roberts AC (2003). Dissociable contributions of the human amygdala and orbitofrontal cortex to incentive motivation and goal selection. J Neurosci 23: 9632-9638.

Babor TF, Higgins-Biddle JC, Sauders JB, Monteiro MG (2001). The Alcohol Use Disorders Identification Test: Guide for Use in Primary Care. World Health Organization: Geneva, Switzerland.

Bechara A (2005). Decision making, impulse control and loss of willpower to resist drugs: A neurocognitive perspective. Nat Neurosci 8: 1458-1463.

Belin D, Jonkman S, Dickinson A, Robbins TW, Everitt BJ (2009). Parallel and interactive learning processes within the basal ganglia: relevance for the understanding of addiction. Behav Brain Res 199: 89-102.

Bierut LJ, Agrawal A, Bucholz KK, Doheny KF, Laurie C, Pugh E, et al, Gene Environment Association Studies (2010). A genomewide association study of alcohol dependence. Proc Natl Acad Sci USA 107: 5082-5087.

Cannon TD, Keller MC (2006). Endophenotypes in the genetic analyses of mental disorders. Annu Rev Clin Psychol 2: 267-290.

Carlson JN, Stevens KD (2006). Individual differences in ethanol self-administration following withdrawal are associated with asymmetric changes in dopamine and serotonin in the medial prefrontal cortex and amygdala. Alcohol Clin Exp Res 30: $1678-1692$.

Cavanna AE, Trimble MR (2006). The precuneus: a review of its functional anatomy and behavioural correlates. Brain 129: 564-583.

Chaudhri N, Sahuque LL, Janak PH (2009). Ethanol seeking triggered by environmental context is attenuated by blocking dopamine D1 receptors in the nucleus accumbens core and shell in rats. Psychopharmacology (Berl) 207: 303-314.

Costa RM (2007). Plastic corticostriatal circuits for action learning: What's dopamine got to do with it? Ann N Y Acad Sci 1104: 172-191.

Crabbe JC, Bell RL, Ehlers CL (2010). Human and laboratory rodent low response to alcohol: is better consilience possible? Addict Biol 15: 125-144.

Critchley HD (2005). Neural mechanisms of autonomic, affective, and cognitive integration. J Comp Neurol 493: 154.

D’Ardenne K, McClure SM, Nystrom LE, Cohen JD (2008). BOLD responses reflecting dopaminergic signals in the human ventral tegmental area. Science 319: 1264-1267.

Deichmann R, Gottfried JA, Hutton C, Turner R (2003). Optimized EPI for fMRI studies of the orbitofrontal cortex. Neuroimage 19: 430-441.

Dick DM, Foroud T (2003). Candidate genes for alcohol dependence: a review of genetic evidence from human studies. Alcohol Clin Exp Res 27: 868-879.

Dick DM, Jones K, Saccone N, Hinrichs A, Wang JC, Goate A et al (2006). Endophenotypes successfully lead to gene identification: results from the collaborative study on the genetics of alcoholism. Behav Genet 36: 112-126.

Filbey FM, Claus E, Audette AR, Niculescu M, Banich MT, Tanabe J et al (2008). Exposure to the taste of alcohol elicits activation of the mesocorticolimbic neurocircuitry. Neuropsychopharmacology 33: 1391-1401.

George MS, Anton RF, Bloomer C, Teneback C, Drobes DJ, Lorberbaum JP et al (2001). Activation of prefrontal cortex and anterior thalamus in alcoholic subjects on exposure to alcoholspecific cues. Arch Gen Psychiatry 58: 345-352.

Goldstein RZ, Craig AD, Bechara A, Garavan H, Childress AR, Paulus MP et al (2009). The neurocircuitry of impaired insight in drug addiction. Trends Cogn Sci 13: 372-380.

Gottesman II, Gould TD (2003). The endophenotype concept in psychiatry: etymology and strategic intentions. Am J Psychiatry 160: 636-645.

Gray MA, Critchley HD (2007). Interoceptive basis to craving. Neuron 54: 183-186.

Grusser SM, Wrase J, Klein S, Hermann D, Smolka MN, Ruf M et al (2004). Cue-induced activation of the striatum and medial 
prefrontal cortex is associated with subsequent relapse in abstinent alcoholics. Psychopharmacology (Berl) 175: 296-302.

Hamann S (2005). Sex differences in the responses of the human amygdala. The Neuroscientist 11: 288-293.

Hansson AC, Rimondini R, Neznanova O, Sommer WH, Heilig M (2008). Neuroplasticity in brain reward circuitry following a history of ethanol dependence. Eur J Neurosci 27: 1912-1922.

Harvey SC, Foster KL, McKay PF, Carroll MR, Seyoum R, Woods JE et al (2002). The GABA(A) receptor alphal subtype in the ventral pallidum regulates alcohol-seeking behaviors. J Neurosci 22: 3765-3775.

Heath AC, Madden PAF, Bucholz KK, Dinwiddie SH, Slutske WS, Bierut LJ et al (1999). Genetic differences in alcohol sensitivity and the inheritance of alcoholism risk. Psychol Med 29: 1069-1081.

Heather N, Booth P, Luce A (1998). Impaired Control Scale: crossvalidation and relationships with treatment outcome. Addiction 93: 761-771.

Heather N, Tebbutt JS, Mattick RP, Zamir R (1993). Development of a scale for measuring impaired control over alcohol consumption: a preliminary report. J Stud Alcohol 54: 700-709.

Hommer DW (1999). Functional imaging of craving. Alcohol Res Health 23: 187-196.

Hutchison KE (2010). Substance use disorders: realizing the promise of pharmacogenomics and personalized medicine. Annu Rev Clin Psychol 6: 577-589.

Hutchison KE, Haughey H, Niculescu M, Schacht J, Kaiser A, Stitzel J et al (2008). The incentive salience of alcohol: translating the effects of genetic variant in CNR1. Arch Gen Psychiatry 65: 841-850.

Ihssen N, Cox WM, Wiggett A, Fadardi JS, Linden DE (2011). Differentiating heavy from light drinkers by neural responses to visual alcohol cues and other motivational stimuli. Cereb Cortex 21: 1408-1415.

Janak PH, Chaudhri N (2010). The potent effect of environmental context on relapse to alcohol-seeking after extinction. Open Addict J 3: 76-87.

Jenkinson M, Bannister P, Brady M, Smith S (2002). Improved optimization for the robust and accurate linear registration and motion correction of brain images. Neuroimage 17: 825-841.

Kalivas PW, Volkow ND (2005). The neural basis of addiction: a pathology of motivation and choice. Am J Psychiatry 162: 1403-1413.

Kareken DA, Claus ED, Sabri M, Dzemidzic M, Kosobud AEK, Radnovich AJ et al (2004). Alcohol-related olfactory cues activate the nucleus accumbens and ventral tegmental area in high-risk drinkers: preliminary findings. Alcohol Clin Exp Res 28: $550-557$.

Kelley AE (2004). Ventral striatal control of appetitive motivation: role in ingestive behavior and reward-related learning. Neurosci Biobehav Rev 27: 765-776.

Knapp CM, Tozier L, Pak A, Ciraulo DA, Kornetsky C (2009). Deep brain stimulation of the nucleus accumbens reduces ethanol consumption in rats. Pharmacol Biochem Behav 92: 474-479.

Knopik VS, Heath AC, Madden PAF, Bucholz KK, Slutske WS, Nelson EC et al (2004). Genetic effects on alcohol dependence risk: re-evaluating the importance of psychiatric and other heritable risk factors. Psychol Med 34: 1519-1530.

Koob GF (2008). A role for brain stress systems in addiction. Neuron 59: 11-34.

Koob GF, Volkow ND (2010). Neurocircuitry of addiction. Neuropsychopharmacology 35: 217-238.

Li CS, Milivojevic V, Constable RT, Sinha R (2005). Recent cannabis abuse decreased stress-induced BOLD signals in the frontal and cingulate cortices of cocaine dependent individuals. Psychiatry Res 140: 271-280.

Lingford-Hughes AR, Daglish MRC, Stevenson BJ, Feeney A, Pandit SA, Wilson SJ et al (2006). Imaging alcohol cue exposure in alcohol dependence using a PET O-15- $\mathrm{H}_{2} \mathrm{O}$ paradigm: results from a pilot study. Addict Biol 11: 107-115.

Lovero KL, Simmons AN, Aron JL, Paulus MP (2009). Anterior insular cortex anticipates impending stimulus significance. Neuroimage 45: 976-983.

McBride D, Barrett SP, Kelly JT, Aw A, Dagher A (2006). Effects of expectancy and abstinence on the neural response to smoking cues in cigarette smokers: an fMRI study. Neuropsychopharmacology 31: 2728-2738.

McBride WJ (2002). Central nucleus of the amygdala and the effects of alcohol and alcohol-drinking behavior in rodents. Pharmacol Biochem Behav 71: 509-515.

McClernon FJ, Kozink RV, Rose JE (2008). Individual differences in nicotine dependence, withdrawal symptoms, and sex predict transient fMRI-BOLD responses to smoking cues. Neuropsychopharmacology 33: 2148-2157.

McClure SM, Daw ND, Montague PR (2003). A computational substrate for incentive salience. Trends Neurosci 26: 423-428.

Marguiles DS, Vincent JL, Kelly C, Lohmann C, Uddin LQ, Biswal BB et al (2009). Precuneus shares intrinsic functional architecture in humans and monkeys. Proc Natl Acad Sci USA 106: 20069-20074.

Meyer-Lindenberg A, Weinberger DR (2006). Intermediate phenotypes and genetic mechanisms of psychiatric disorders. Nat Rev Neurosci 7: 818-827.

Myrick H, Anton RF, Li X, Henderson S, Randall PK, Voronin K (2008). Effect of naltrexone and ondansetron on alcohol cueinduced activation of the ventral striatum in alcohol-dependent people. Arch Gen Psychiatry 65: 466-475.

Myrick H, Anton RF, Li XB, Henderson S, Drobes D, Voronin K et al (2004). Differential brain activity in alcoholics and social drinkers to alcohol cues: relationship to craving. Neuropsychopharmacology 29: 393-402.

Naqvi NH, Bechara A (2009). The hidden island of addiction: the insula. Trends Neurosci 32: 56-67.

Naqvi NH, Rudrauf D, Damasio H, Bechara A (2007). Damage to the insula disrupts addiction to cigarette smoking. Science 315: 531-534.

Paulus MP (2007). Decision-making dysfunctions in psychiatryaltered homeostatic processing? Science 318: 602-606.

Robinson TE, Berridge KC (2003). Addiction. Ann Rev Psychol 54: 23-53.

Rodd ZA, Bell RL, Sable HJK, Murphy JM, McBride WJ (2004). Recent advances in animal models of alcohol craving and relapse. Pharmacol Biochem Behav 79: 439-450.

Rushworth MFS, Walton ME, Kennerley SW, Bannerman DM (2004). Action sets and decisions in the medial frontal cortex. Trends Cogni Sci 8: 410-417.

Savine AC, Braver TS (2010). Motivated cognitive control: reward incentives modulate preparatory neural activity during taskswitching. J Neurosci 30: 10294-10305.

Schneider F, Habel U, Wagner M, Franke P, Salloum JB, Shah NJ et al (2001). Subcortical correlates of craving in recently abstinent alcoholic patients. Am J Psychiatry 158: 1075-1083.

Schultz W (2007). Multiple dopamine functions at different time courses. Annu Rev Neurosci 30: 259-288.

Sharma D, Albery IP, Cook C (2001). Selective attentional bias to alcohol related stimuli in problem drinkers and non-problem drinkers. Addiction 96: 285-295.

Skinner HA, Horn JL (1984). Alcohol Dependence Scale: Users Guide. Addiction Research Foundation: Toronto, Canada.

Smith SM, Jenkinson M, Woolrich MW, Beckmann CF, Behrens $\mathrm{TE}$, Johansen-Berg $\mathrm{H}$ et al (2004). Advances in functional and structural MR image analysis and implementation as FSL. Neuroimage 23(Suppl 1): S208-S219.

Sobell LC, Sobell MB (1992). Timeline follow-back: a technique for assessing self-reported alcohol consumption. In: Litten RZ, Allen JP (eds). Measuring Alcohol Consumption: Psycho- 
social and Biochemical Methods. Humana Press, Totowa, NJ, pp 41-72.

Stetter F, Ackermann K, Bizer A, Straube ER, Mann K (1995). Effects of disease-related cues in alcoholic inpatients: results of a controlled 'Alcohol Stroop' study. Alcohol Clin Exp Res 19: 593-599.

Stormark KM, Field NP, Hugdahl K, Horowitz M (1997). Selective processing of visual alcohol cues in abstinent alcoholics: an approach-avoidance conflict? Addict Behav 22: 509-519.

Stuber GD, Hopf FW, Hahn J, Cho SL, Guillory A, Bonci A (2008). Voluntary ethanol intake enhances excitatory synaptic strength in the ventral tegmental area. Alc Clin Exp Res 32: 1714-1720.

Sullivan JT, Sykora K, Schneiderman J, Naranjo CA, Sellers EM (1989). Assessment of alcohol withdrawal: the revised Clinical Institute Withdrawal Assessment for Alcohol Scale (CIWA-Ar). Br J Addict 84: 1353-1357.

Tapert SF, Brown GG, Baratta MV, Brown SA (2004). fMRI BOLD response to alcohol stimuli in alcohol dependent young women. Addict Behav 29: 33-50.

Treutlein J, Cichon S, Ridinger M, Wodarz N, Soyka M, Zill P et al (2009). Genome-wide Association Study of Alcohol Dependence. Arch Gen Psychiatry 66: 773-784.

Vollstadt-Klein S, Wichert S, Rabinstein J, Buhler M, Klein O, Ende G, et al, Mann K (2010). Initial, habitual and compulsive alcohol use is characterized by a shift of cue processing from ventral to dorsal striatum. Addiction 105: 1741-1749.
Wang J, Carnicella S, Phamluong K, Jeanblanc J, Ronesi JA, Chaudhri $\mathrm{N}$ et al (2007). Ethanol induces long-term facilitation of NR2B-NMDA receptor activity in the dorsal striatum: implications for alcohol drinking behavior. J Neurosci 27: 3593-3602.

Wickens J (1997). Basal ganglia: structure and computations. Network: Computation in Neural Systems 8: R77-R109.

Wilson SJ, Sayette MA, Fiez JA (2004). Prefrontal responses to drug cues: a neurocognitive analysis. Nat Neurosci 7: 211-214.

Wise RA (2009). Roles for nigrostriatal - not just mesocorticolimbic-dopamine in reward and addiction. Trends Neurosci 32: 517-524.

Woolrich MW, Behrens TE, Beckmann CF, Jenkinson M, Smith SM (2004). Multilevel linear modelling for FMRI group analysis using Bayesian inference. Neuroimage 21: 1732-1747.

Worsley KJ, Evans AC, Marrett S, Neelin P (1992). A threedimensional statistical analysis for CBF activation studies in human brain. J Cereb Blood Flow Metab 12: 900-918.

Worsley KJ, Marrett S, Neelin P, Vandal AC, Friston KJ, Evans AC (1996). A unified statistical approach for determining significant signals in images of cerebral activation. Hum Brain Mapp 4: 58-73.

Wrase J, Schlagenhauf F, Kienast T, Wustenberg T, Bermpohl F, Kahnt $\mathrm{T}$ et al (2007). Dysfunction of reward processing correlates with alcohol craving in detoxified alcoholics. Neuroimage 35: 787-794.

Supplementary Information accompanies the paper on the Neuropsychopharmacology website (http://www.nature.com/npp) 